Use of ther mal I y anneal ed mil ti l ayer gol d nanoparti cle fil ms i $\mathrm{n}$ combi nat i on anal ysi s of l ocal i zed surface pl asmon resonance sensi ng and MALD mass spect romet ry

\begin{tabular}{|l|l|}
\hline 著者 & $\begin{array}{l}\text { I nut a Nasayuki, Ar akawa Ryui chi , Kawasaki } \\
\text { H deya }\end{array}$ \\
\hline $\begin{array}{l}\text { j our nal or } \\
\text { publ i cat i on t i t l e }\end{array}$ & Anal yst \\
\hline vol une & 136 \\
\hline page range & $1167-1176$ \\
\hline year & 2011 \\
\hline URL & ht t p: //hdl . handl e. net /10112/5862 \\
\hline
\end{tabular}




\title{
Use of thermally annealed multilayer gold nanoparticle films in combination analysis of localized surface plasmon resonance sensing and MALDI mass spectrometry $\dagger$
}

\author{
Masayuki Inuta, Ryuichi Arakawa and Hideya Kawasaki* \\ Received 26th October 2010, Accepted 6th January 2011 \\ DOI: 10.1039/c0an00826e
}

\begin{abstract}
A self-assembled film of gold nanoparticles (AuNPs) with a raspberry-like morphology was prepared on a glass plate by the layer-by-layer thermal annealing of multilayer films of AuNPs. It was possible to control the morphology of the obtained films of AuNPs by changing the annealing temperature, duration of annealing, and number of layers. On investigating the plasmonic properties of these films, we found that AuNP films with a raspberry-like morphology yielded the highest refractive index unit, which is a critical parameter in localized surface plasmon resonance (LSPR) sensing, as compared to other types of AuNP films. Self-assembled AuNP films with a raspberry-like morphology were subsequently functionalized with 11-mercaptoundecanoic acid (MUA) to enable the binding of lysozyme to the MUA-modified Au surface. The superior limit of detection for the LSPR sensing of lysozyme in a buffer solution was found to be in the picomolar range $\left(\sim 10^{-12} \mathrm{M}\right)$. The high sensitivity observed in the region was attributed to the raspberry-like morphology, where the AuNPs were packed closely together, and the electromagnetic field confinement was most intense (i.e., at hot spots). The MUA-modified, self-assembled AuNP films with a raspberry-like morphology were finally used in the combination analysis of LSPR sensing and matrix-assisted laser desorption/ionization mass spectrometry (MALDI-MS) for the selective detection and identification of lysozyme in human serum.
\end{abstract}

\section{Introduction}

Noble metal nanoparticles such as gold nanoparticles (AuNPs) and silver nanoparticles have unique optical properties owing to the localized surface plasmon resonance (LSPR) phenomenon, which is a collective oscillation of conduction electrons excited by electromagnetic radiation. LSPR gives rise to intense surface plasmon adsorption bands in the visible to the near-IR region in the optical spectrum. ${ }^{1-6}$ The absorption maximum and intensity of the LSPR bands depend on the size and shape of the metal $\mathrm{NPs}$, the distance between them, and the effective refractive index of the local environment. ${ }^{1-9}$ Among these properties, the dependence of LSPR bands on the change in the local refractive index around NPs is associated with the use of NPs in label-free sensing applications, where the LSPR sensing is based on the change in the local refractive index around NPs, caused by analyte binding. This LSPR wavelength shift, which is based on

Department of Chemistry and Materials Engineering, Faculty of Chemistry, Materials and Bioengineering, Kansai University, 3-3-35 Yamate-cho, Suita-shi, Osaka, 564-8680, Japan. E-mail: hkawa@, kansai-u.ac.jp; Fax: +81 (6)6339 4026; Tel: +81 (6)6368 5647

$\uparrow$ Electronic supplementary information (ESI) available: The carefully chosen points for the preparation of LbL film and the LSPR sensing and UV-vis spectrum of a raspberry-like assembly of AuNP films. See DOI: $10.1039 / \mathrm{c} 0 \mathrm{an} 00826 \mathrm{e}$ the change in the refractive index, can be examined by using UV-vis transmission or reflection spectroscopy. Several types of LSPR sensing platforms have been reported such as substrates on which immobilized NPs from a colloidal solution, ${ }^{10-18}$ lithographically fabricated triangular-nanocrystal arrays, ${ }^{19-28}$ nanohole arrays produced by ion-beam lithography, ${ }^{29-32}$ and transparent substrates on which metal island films are fabricated by vapor deposition. ${ }^{33-39}$ When LSPR sensing is conducted in transmission mode, it is known as transmission localized surface plasmon resonance (T-LSPR) sensing. The T-LSPR sensing has two main advantages: it requires a simple experimental setup and is inexpensive to perform. One of the platforms used in T-LSPR is the immobilized AuNPs on a substrate from a colloidal solution; the entire process of making such a substrate is simple and requires only common laboratory supplies and equipment. ${ }^{10-18}$ Various anisotropic NPs such as rod-shaped or triangular NPs were utilized in T-LSPR sensing owing to their high LSPR sensitivity. Extensive studies have been conducted on LSPR sensing; however, further improvements are required, e.g., better detection limits, higher stability of metal NPs on the substrate, and good reproducibility of the LSPR platform. In addition, the combination of LSPR with other techniques for the identification of analytes in complex mixtures remains an important challenge. ${ }^{9}$ Because the LSPR signal depends only on the change in local refractive index around the metal nanoparticles, the 
identification of analytes can be achieved only by using specific biomolecular recognition techniques that involve the use of antibodies. The use of the combination of LSPR sensing with laser desorption/ionization mass spectrometry for the identification of analytes has been recently reported. ${ }^{40}$

In this paper, we discuss the morphological changes induced by the layer-by-layer ( $\mathrm{LbL}$ ) thermal annealing of multilayer films of AuNPs on a glass substrate and the effects of these morphological changes on the plasmonic properties of the films. We found that a raspberry-like self-assembly of AuNPs (i.e., clustering of AuNPs) occurred during the thermal annealing of LbL films of AuNPs. The resultant self-assembled AuNP film with a raspberry-like morphology is suitable for use in T-LSPR sensing. Finally, we present the combination analysis of LSPR sensing and matrix-assisted laser desorption/ionization mass spectrometry (MALDI-MS) for the selective detection and identification of lysozyme (Lyz) in human serum using a selfassembled AuNP film with a raspberry-like morphology.

\section{Experimental}

\section{Materials}

Tetrachloroauric acid $\left(\mathrm{HAuCl}_{4}\right)$ and triammonium citrate were obtained from Wako Chemical Co. (Osaka, Japan). $\alpha$-Cyano-4hydroxycinnamic acid (CHCA) and lysozyme (Lyz) were purchased from Sigma-Aldrich (St Louis, MO). Human serum was purchased from Chemicon International Inc. (CA, USA). 11-Mercaptoundecanoic acid (MUA) was obtained from Tokyo Chemical Industries Ltd (Tokyo, Japan). Poly(allylamine hydrochloride) (PAH, $\left.M_{\mathrm{w}}=70000\right)$ was purchased from SigmaAldrich.

\section{Synthesis of triammonium citrate-capped AuNPs}

Triammonium citrate-capped AuNPs were prepared according to our previous study. ${ }^{41}$ An aqueous solution $(1 \mathrm{~mL})$ of tetrachloroauric acid $(24.3 \mathrm{mM})$ and an aqueous solution $(1 \mathrm{~mL})$ of triammonium citrate $(80 \mathrm{mM})$ were added rapidly to refluxing water $(99 \mathrm{~mL})$. The solution was heated under reflux for an additional $30 \mathrm{~min}$. During this period, the color of the solution changed from pale yellow to wine red. The solution was allowed to cool to room temperature. The diameters of the AuNPs were estimated to be approximately $20-30 \mathrm{~nm} .{ }^{41}$

\section{Preparation of LbL films}

A plain glass plate from Matsunami Co. (Osaka, Japan) was used as a substrate. A layer-by-layer ( $\mathrm{LbL}$ ) approach was used to fabricate the AuNP films, i.e., a positively charged polymer and negatively charged AuNPs were alternately deposited onto the substrate. The AuNPs were negatively charged because of the stabilizing layer of triammonium citrate, and therefore, a positively charged polyelectrolyte polymer, PAH, was chosen as an LbL partner. Prior to any modification, the glass plate was cleaned by immersing it in a freshly prepared $5: 1: 1 \mathrm{vol} \%$ mixture of water, hydrogen peroxide, and $29 \%$ ammonia solution at $70{ }^{\circ} \mathrm{C}$ for $30 \mathrm{~min}$. The LbL assembly of AuNPs was obtained by the sequential dipping of the plate in an aqueous solution $(10 \mathrm{~mL})$ of PAH $\left(3 \mathrm{mg} \cdot \mathrm{mL}^{-1}\right)$ for $60 \mathrm{~min}$ and an aqueous solution $(10 \mathrm{~mL})$ of AuNPs for $24 \mathrm{~h}$. After each dip, the substrate was gently rinsed twice with pure water. In this manner, we prepared multilayer films, $(\mathrm{AuNP} / \mathrm{PAH})_{n}(n=1$ and 3$)$, using the LbL method. Here, the number " $n$ " does not indicate the number of AuNP layers but the number of deposition cycles. That is, we define it as follows: $n=1$ is glass-(PAH-AuNP), and $n=3$ is glass-(PAH-AuNP)-(PAH-AuNP)-(PAH-AuNP). The last layer is AuNP for MALDI experiments.

The thermal annealing of (AuNP/PAH $)_{n}$ films was carried out in air for a different temperature range of 300 to $500{ }^{\circ} \mathrm{C}$ in an electronic oven (KDF S-70). The annealing duration was changed from 1 to $5 \mathrm{~h}$. The heating rate was $8^{\circ} \mathrm{C} \mathrm{min}-1$, and the annealed substrate was left to cool to room temperature in the air.

Immobilized AuNPs on the glass substrate after the thermal annealing were chemically modified by the formation of a selfassembled monolayer (SAM) of 11-mercaptoundecanoic acid (MUA). The gold nanoparticle-deposited substrate was immersed in ethanol once, and then incubated in a $50 \mathrm{mM}$ solution of MUA in ethanol for $24 \mathrm{~h}$ at room temperature. The substrate was rinsed with ethanol. Even though high concentration of MUA and long immersing time were employed, the detachment of AuNPs on the glass substrate did not occur. The MUA modification increased the absorbance and a red shift with $5 \mathrm{~nm}$ was observed for the LSPR maximum, as shown in the ESI $\uparrow$. By the excess amount of surface modification of MUA compared to $\mathrm{PAH}$, it is likely that the surface charges are negatively charged as a whole. The negatively charged surface by MUA can cause the selective detection of positively charged Lyz from human serum.

The quality of these LbL films as well as the reproducibility of the preparation process is important, together with the results obtained in the LSPR sensing. The several points were chosen to obtain better reproducibility of this methodology. The careful points for the preparation of LbL film and the LSPR sensing are described in the ESI $\uparrow$.

\section{MALDI-MS}

MALDI mass spectra were acquired in the linear mode using an AXIMA-CFR time-of-flight mass spectrometer (Shimadzu/Kratos, Manchester, UK) with a pulsed nitrogen laser $(\lambda=337 \mathrm{~nm})$. One hundred laser shots were used to acquire the mass spectra. The analyte ions were accelerated at $20 \mathrm{kV}$ under delayed extraction conditions. LbL multilayer films composed of AuNPs on the glass plate were used in the MALDIMS with a CHCA matrix.

\section{Atomic force microscopy (AFM)}

AFM images for the substrates were acquired at $25 \pm 2{ }^{\circ} \mathrm{C}$ using a NanoScope IIIa (Veeco) for tapping modes. The AFM images were obtained at a scan rate of $0.5 \mathrm{~Hz}$ using silicon tips with a nominal spring constant of $42 \mathrm{~N} \mathrm{~m}^{-1}$ in the air.

\section{UV-visible spectroscopy}

UV-vis spectra were obtained on a JASCO Ubest-670 UV-Vis spectrophotometer with a temperature controller. Glass slides coated with the multilayer films were placed perpendicular to the 
beam. The measurements were performed using the specialized cell and the holder, as shown in the ESI†.

\section{Thermo gravimetric (TG) analysis}

TG analysis was carried out using a TG-DTA Thermo plus EVO (Rigaku). Approximately, $10 \mathrm{mg}$ of PAH was weighed on an alumina crucible and isothermally heated to $30{ }^{\circ} \mathrm{C}$ for $10 \mathrm{~min}$ under the air flow and then heated from 30 to $450{ }^{\circ} \mathrm{C}$; this included a holding temperature of $300^{\circ} \mathrm{C}$ for $1 \mathrm{~h}$ in a static air atmosphere. The heating rate was $8{ }^{\circ} \mathrm{C} \mathrm{min}^{-1}$. The procedure for heating was the same as the thermal annealing process used for obtaining the self-assembled AuNP films with a raspberry-like morphology. Indium was used as a reference.

\section{Combination analysis of LSPR sensing and MALDI-MS for detection of lysozyme in human serum}

Human serum containing Lyz $\left(10^{-4}\right.$ or $\left.10^{-5} \mathrm{~g} \mathrm{~mL}^{-1}\right)$ was prepared. A self-assembled AuNP film with a raspberry-like morphology (i.e., (AuNP/PAH) $)_{3}$ film annealed at $300{ }^{\circ} \mathrm{C}$ for $1 \mathrm{~h}$ ) was immersed in the serum solution for $1 \mathrm{~h}$. This AuNP film, to which Lyz was bound, was then washed thrice with water; this was followed by LSPR sensing conducted in the air and a MALDI-MS. In the MALDI-MS, a $1 \mu \mathrm{L}$ solution of CHCA matrix (20 mg mL $\mathrm{mL}^{-1}, 3: 7$ water-methanol mixture) was further applied to the AuNP film to which Lyz was bound; the film was dried at room temperature before conducting MS.

\section{Results and discussion}

\section{Morphology and LSPR properties of LbL multilayer films of AuNPs}

LbL multilayer films of AuNPs were prepared on a glass substrate and then annealed in the air. The control of the morphology and optical properties was achieved by varying the number of layers in the LbL film, annealing temperature, and duration of annealing. For a comparison, a film of randomly aggregated AuNPs on a glass substrate was obtained by adding $\mathrm{NaCl}$ to a colloidal solution of AuNPs.

In the case of a monolayer of AuNPs and poly(allylamine hydrochloride), i.e., (AuNP/PAH) 1 , AFM image of the deposited AuNPs prior to thermal annealing mostly revealed individual nanoparticles on the glass surface (Fig. 1a); however, some partial aggregates of AuNPs were also observed. The protrusions extending from the surface were approximately $30 \mathrm{~nm}$ long. This value was almost consistent with the diameter of the AuNPs used. On the other hand, the lateral directions of NPs in the AFM image were significantly larger in length than in reality because of the convolution of the AFM tip. The thermal annealing of a monolayer (AuNP/PAH) $)_{1}$ film at $300{ }^{\circ} \mathrm{C}$ and 500 ${ }^{\circ} \mathrm{C}$ for $1 \mathrm{~h}$ caused an increase, via the thermal-sintering or fusion process, in the average diameter of the individual nanoparticles from $\sim 60 \mathrm{~nm}$ (before annealing) to $\sim 100 \mathrm{~nm}\left(300^{\circ} \mathrm{C}\right)$ and $\sim 120$ $\mathrm{nm}\left(500{ }^{\circ} \mathrm{C}\right)($ Fig. 1a-c). The interparticle distance of AuNPs increased during the thermal annealing process, resulting in more dispersed AuNPs on the plate at $500{ }^{\circ} \mathrm{C}$. The UV-vis spectra confirmed these morphological changes in thermally annealed AuNPs (Fig. 2a). The LSPR peak for unannealed AuNPs appeared at around $520 \mathrm{~nm}$ with a shoulder peak at around $615 \mathrm{~nm}$ due to the partial aggregation of AuNPs. After thermal annealing at $500{ }^{\circ} \mathrm{C}$, the shoulder peak disappeared, and a single large LSPR peak because of the dispersed AuNPs was observed at around $534 \mathrm{~nm}$, indicating that the spectra data were in good agreement with the AFM observations.

The unannealed multilayer (AuNP/PAH) $)_{3}$ film showed more densely packed AuNPs on the glass plate owing to the multiple deposition (Fig. 1d). Clustering of AuNPs was formed during the LbL process; they can be seen in the expanded AFM images (Fig. 3a), where it appears that the AuNPs in the multilayer films do not fuse to form larger particles. We examined the thermally annealed multilayer $(\mathrm{AuNP} / \mathrm{PAH})_{3}$ film. Clearly, the morphology of the annealed film was different from that of the unannealed film, as shown in the AFM images (Fig. 3b and c). When the film was annealed at $300{ }^{\circ} \mathrm{C}$ for $1 \mathrm{~h}$, the individual nanoparticles partially fused to form closely packed clusters of AuNPs, as seen in the expanded AFM image (Fig. 3b). Hereafter, we refer to this formation as "a self-assembled film of AuNPs with a raspberry-like morphology". When annealing was carried out at a higher temperature of $500{ }^{\circ} \mathrm{C}$ for $1 \mathrm{~h}$, the clusters of AuNPs disappeared due to further fusion events, and larger AuNPs with sizes of approximately $200 \mathrm{~nm}$ formed on the glass plate (Fig. 3c). The TG analysis data showed that the cationic PAH polymer required a high temperature of at least $450{ }^{\circ} \mathrm{C}$ for complete mass loss to occur (Fig. 4), which, in turn, would cause the cationic polymer to be burned out at $500{ }^{\circ} \mathrm{C}$. Subsequently, increasing van der Waals interactions among the deprotected AuNPs would result in the direct contact between AuNPs. On the other hand, the cationic polymer may still exist during thermal annealing at $300{ }^{\circ} \mathrm{C}$ for $1 \mathrm{~h}$, resulting in the slow and partial fusion of adjacent nanoparticles to form clusters of AuNPs on account of the limited mobility of AuNPs covered with the surviving cationic PAH polymer. Therefore, it is likely that the raspberry-like morphology of AuNPs on the glass plate can be obtained via the slow and partial fusion of individual nanoparticles in the $(\mathrm{AuNP} / \mathrm{PAH})_{3}$ film. It should be noted that longer annealing durations of $3 \mathrm{~h}$ or $5 \mathrm{~h}$ resulted in the further fusion of AuNPs even at $300^{\circ} \mathrm{C}$, and finally, in the disappearance of the raspberry-like morphology (not shown).

The UV-vis spectra for multilayer $(\mathrm{AuNP} / \mathrm{PAH})_{3}$ films were consistent with the AFM observations (Fig. 2b). When the number of layers was increased to three, the LSPR absorption band moved toward longer wavelengths (i.e., it red shifted) and showed broadening. An intense band at around $780 \mathrm{~nm}$ was dominant for the $(\mathrm{AuNP} / \mathrm{PAH})_{3}$ multilayer films. It is likely that the intense SPR band at around $780 \mathrm{~nm}$ originated from additional interactions between the AuNPs located in adjacent layers (interlayer and interparticle coupling). ${ }^{41}$ However, the broadening of the LSPR peak is unsuitable during LSPR sensing. We, therefore, did not use the unannealed $(\mathrm{AuNP} / \mathrm{PAH})_{3}$ film for LSPR sensing. On the other hand, when the $(\mathrm{AuNP} / \mathrm{PAH})_{3}$ multilayer film was annealed at $300^{\circ} \mathrm{C}$ for $1 \mathrm{~h}$, the AuNPs started fusing. As a result, the intensity of the SPR band at around $780 \mathrm{~nm}$ decreased, and the spectrum showed a relatively sharp LSPR absorption band at around $570 \mathrm{~nm}$, owing to the partial fusion of individual nanoparticles into closely packed clusters; this fusion resulted in the formation of a self-assembled AuNP film with a raspberry-like morphology. It should be noted that 

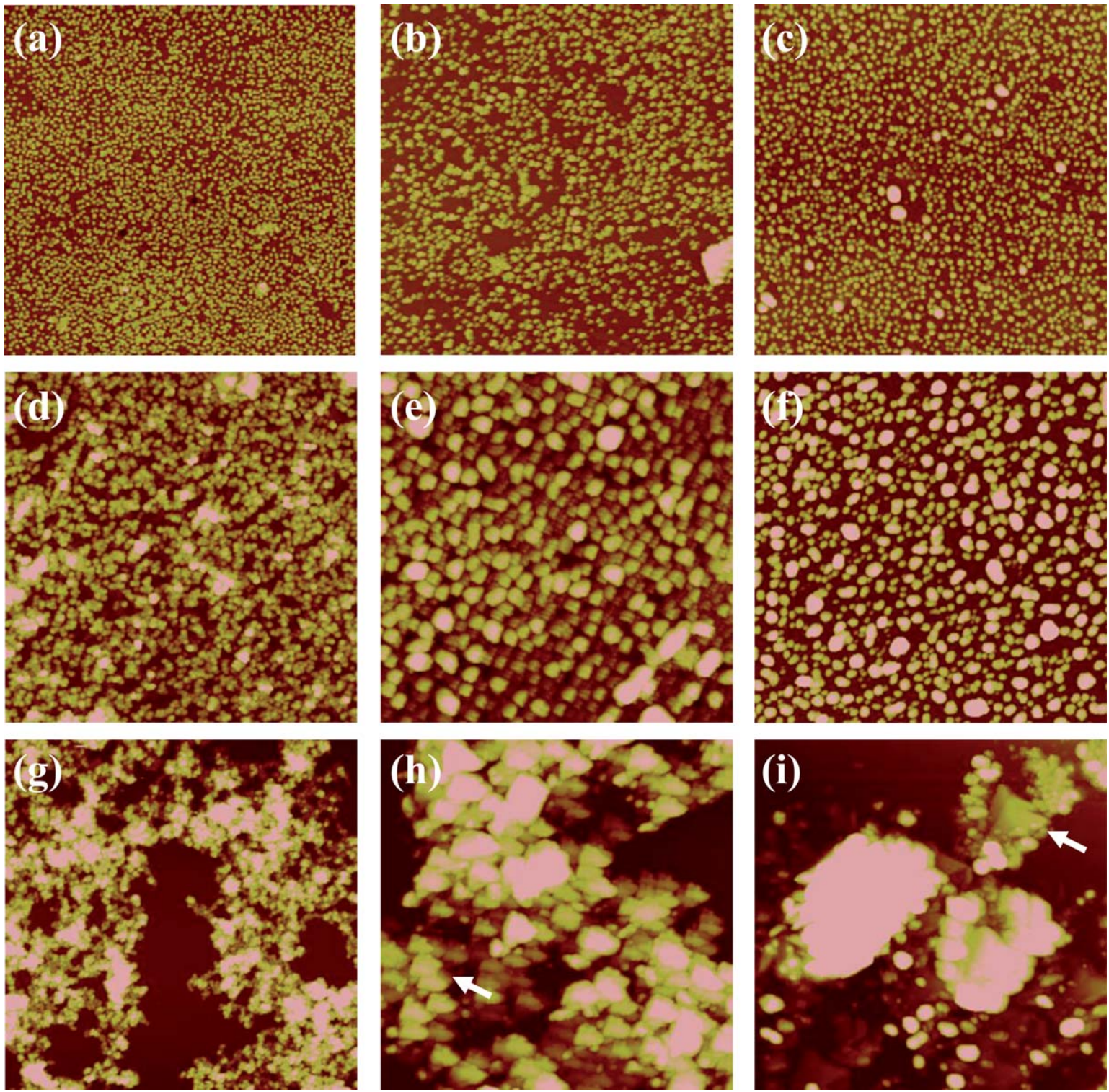

Fig. 1 AFM images of LbL multilayer (AuNP/PAH) films $(5 \mu \mathrm{m} \times 5 \mu \mathrm{m})$ : (a) $n=1$, before annealing, (b) $n=1$, after annealing at $300{ }^{\circ} \mathrm{C}$ for $1 \mathrm{~h}$, (c) $n=1$, after annealing at $500{ }^{\circ} \mathrm{C}$ for $1 \mathrm{~h}$, (d) $n=3$, before annealing, (e) $n=3$, after annealing at $300{ }^{\circ} \mathrm{C}$ for $1 \mathrm{~h}$, and (f) $n=3$, after annealing at $500{ }^{\circ} \mathrm{C}$ for $1 \mathrm{~h}$. The AFM images of random aggregates of AuNPs induced by addition of $300 \mathrm{mM} \mathrm{NaCl}(5 \mu \mathrm{m} \times 5 \mu \mathrm{m})$ : (g) before annealing, (h) after annealing at $300{ }^{\circ} \mathrm{C}$ for $1 \mathrm{~h}$, and (i) after annealing at $500{ }^{\circ} \mathrm{C}$ for $1 \mathrm{~h}$.

the raspberry-like islands of AuNPs were not formed over the whole surface. The ratio of gold raspberry-like islands on the surface was from 40 to $60 \%$ in $5 \mu \mathrm{m} \times 5 \mu \mathrm{m}$ area of the AFM image. The number distribution of AuNPs within the islands was broad in the range of 5-20 from batch to batch.

As a result, the measurements at different points in the surface of the substrate may affect the LSPR peak (maximum wavelength and the width). To perform the measurements in the same substrate position, we prepared a custom-order quartz cell and the cell holder to fix it to the same position in the UV-vis spectrometer, as shown in the ESI $\dagger$. On the other hand, it is considered that the inhomogeneities with micrometre scale observed in AFM images are averaged in the LSPR sensing, since the optically illuminated size on the sample surface in the UV-vis spectrometer is $1.1 \mathrm{~cm}$ long and $0.15 \mathrm{~cm}$ wide. As a result, the
LSPR peaks provide cumulative information about a large area of the sample surface.

For comparison, a film of randomly aggregated AuNPs on a glass substrate was also obtained by adding $300 \mathrm{mM} \mathrm{NaCl}$ to a colloidal solution of AuNPs (Fig. 1g). After thermal annealing at $300^{\circ} \mathrm{C}$ or $500^{\circ} \mathrm{C}$ for $1 \mathrm{~h}$, the film had an irregular morphology, and it was difficult to control the shape of the aggregated AuNPs that formed during the thermal annealing (Fig. $1 \mathrm{~h}$ and i, respectively).

In the case of the AuNPs that aggregated because of $\mathrm{NaCl}$ addition, it is possible that the AuNPs were highly mobile in the absence of the cationic PAH polymer during thermal annealing in contrast to those in the LbL multilayer film whose mobility was possibly hampered by the presence of the cationic polymer. Thus, it is likely, in the former case, that the AuNPs diffused 

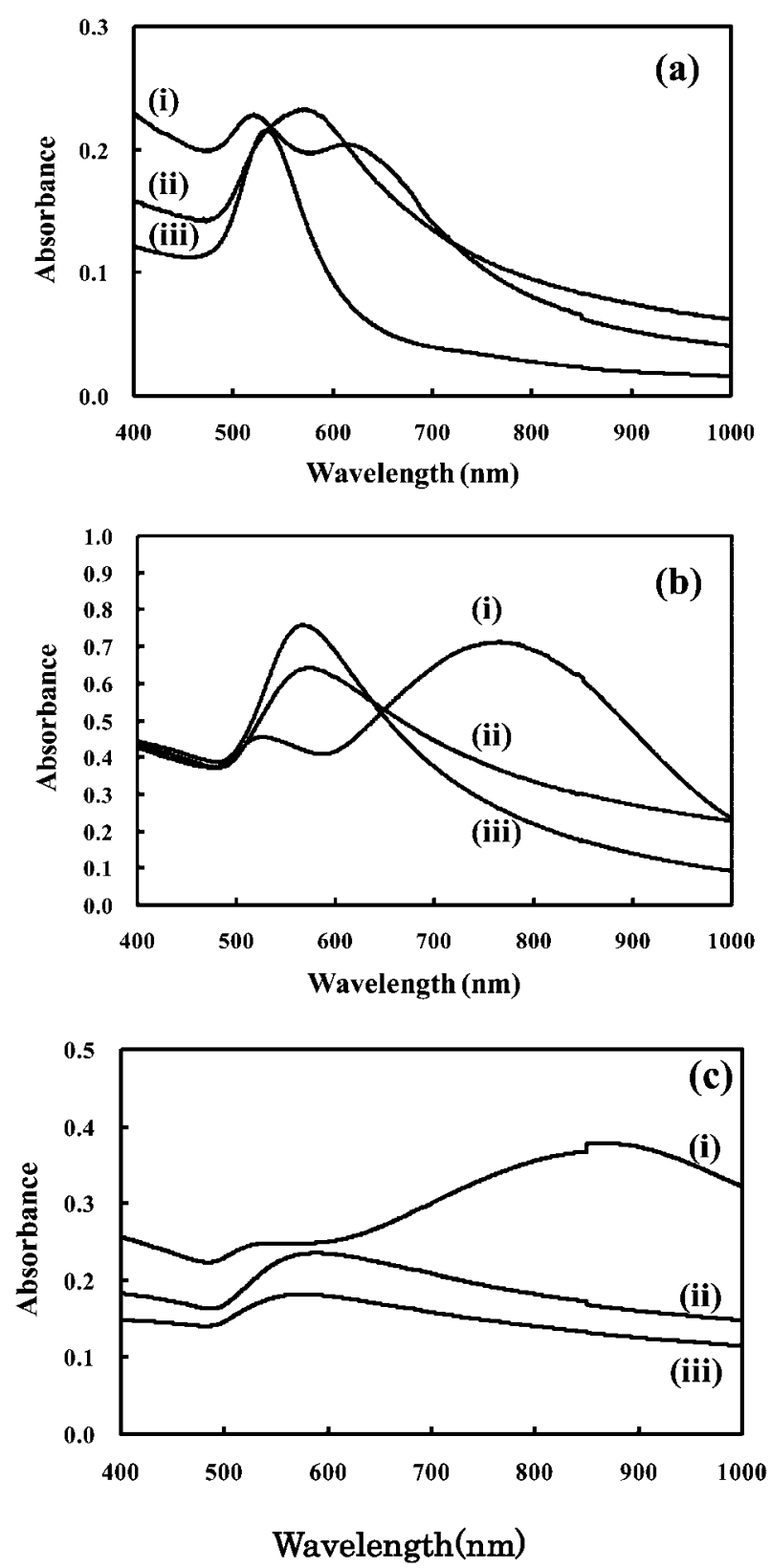

Fig. 2 (a) UV-vis spectra of monolayer (AuNP/PAH) $)_{1}$ films in water: (i) before annealing, (ii) after annealing at $300{ }^{\circ} \mathrm{C}$ for $1 \mathrm{~h}$, and (iii) after annealing at $500{ }^{\circ} \mathrm{C}$ for $1 \mathrm{~h}$. (b) UV-vis spectra of multilayer (AuNP/ $\mathrm{PAH})_{3}$ films: (i) before annealing, (ii) after annealing at $300{ }^{\circ} \mathrm{C}$ for $1 \mathrm{~h}$, and (iii) after annealing at $500{ }^{\circ} \mathrm{C}$ for $1 \mathrm{~h}$. (c) UV-vis spectra of random aggregates of AuNPs induced by addition of $300 \mathrm{mM} \mathrm{NaCl}$ : (i) before annealing, (ii) after annealing at $300^{\circ} \mathrm{C}$ for $1 \mathrm{~h}$, and (iii) after annealing at $500{ }^{\circ} \mathrm{C}$ for $1 \mathrm{~h}$.

rapidly to form AuNP aggregates, and then fused to form larger nanoparticles.

It is noteworthy that triangular AuNPs were observed at random intervals after the thermal annealing (as denoted by arrows in Fig. 1h and i). It has been reported that thermal annealing can result in the atomic rearrangement of metal nanoparticles and convert the internal particle structure from a disordered phase to an ordered phase. ${ }^{42}$ It was also found that the thermal annealing of AuNPs induces atomic rearrangement, thereby producing predominant (111) facets $;^{43}$ the high-affinity adsorption of $\mathrm{Cl}^{-}$ions onto gold surfaces was also reported. ${ }^{44}$ These two effects - the annealing-induced predominant (111) facets and the high-affinity adsorption of $\mathrm{Cl}^{-}$ions from $\mathrm{NaCl}-$ could be responsible for producing triangular AuNPs with predominant (111) facets. However, because of the significant broadening of the LSPR peak, the random aggregates of AuNPs, induced by the addition of $\mathrm{NaCl}$, were unsuitable for use in LSPR sensing (Fig. 2c).

\section{Bulk refractive index unit of annealed (AuNP/PAH) ${ }_{n}$ films}

In LSPR sensing, the refractive index sensitivity (RIS) or refractive index unit (RIU) is a critical parameter., ${ }^{7,8}$ RIU and RIS are usually determined by measuring the shift of the LSPR peak upon the immersion of the substrate in different solvents having variable refractive indexes. The RIU value, defined as the LSPR wavelength shift $\left(\lambda_{\text {shift }}\right)$ per refractive index change of the surrounding medium, i.e., $\left(\Delta \lambda_{\text {shift }} / \Delta n\right)$, was evaluated for annealed $(\mathrm{AuNP} / \mathrm{PAH})_{1}$ and $(\mathrm{AuNP} / \mathrm{PAH})_{3}$ films by measuring the UV-vis spectra of these films in various solvents such as water, 1-propanol, ethylene glycol, glycerin, benzene, and benzaldehyde, having refractive indexes in the range of 1.333-1.546. The increase in the refractive index of the solvent leads to a red shift in the LSPR band, as shown by the typical examples in Fig. 5a and $\mathrm{b}$. This red shift is in agreement with the MaxwellGarnett effective medium theory. ${ }^{45}$ The LSPR wavelength shift showed a linear dependence on the refractive index of the surrounding solvent (Fig. 5c), and we estimated the RIU sensitivity of various annealed AuNP films from the slope $\left(\Delta \lambda_{\text {shift }} / \Delta n\right)$. We replicated the measurements three times using three different substrates. The variability in the LSPR wavelength shift was up to $\pm 3 \mathrm{~nm}$ for the case of those measured in various solvents. The RIU values for these $(\mathrm{AuNP} / \mathrm{PAH})_{n}$ films provided comparable results for evaporated $\mathrm{Au}$ island films on substrates (60-120 nm per RIU). ${ }^{39}$ The RIU value (125 nm per RIU) of the (AuNP/ $\mathrm{PAH})_{3}$ film annealed at $300{ }^{\circ} \mathrm{C}$ for $1 \mathrm{~h}$ was the highest among all the annealed (AuNP/PAH $)_{n}$ films examined in this study.

For single-component nanoparticles with sizes of less than $130 \mathrm{~nm}$, the theoretical consideration of the LSPR sensitivity (i.e., RIU sensitivity) revealed that there was a linear relationship between the RIU values and the LSPR peak wavelength, regardless of the structure of nanoparticles, such as nanodisk and cylindrical. ${ }^{7,8}$ Further, in the case of the annealed (AuNP/PAH) films examined in this study, the RIU values were found to have an almost linear dependence on the LSPR peak wavelength. This dependence was observed irrespective of the differences in the size and morphology of the AuNP films (Fig. 5d), except for the self-assembled AuNP films with a raspberry-like morphology $\left(n=3\right.$, annealed at $300{ }^{\circ} \mathrm{C}$ for $1 \mathrm{~h}$ ). It should be noted that increasing the duration of annealing at $300{ }^{\circ} \mathrm{C}$ from $1 \mathrm{~h}$ to $5 \mathrm{~h}$ for the AuNP films with a raspberry-like morphology resulted in the disappearance of the raspberry-like morphology and a decrease in the RIU sensitivity (RIU $\approx 90$ ).

In measurements on LSPR wavelength shift in various solvents (Fig. 5c), we used an identical glass substrate to reduce the variability from substrate to substrate. On immersing the glass slides into various solvents, the detachment of AuNPs did not occur. The immobilized AuNPs on the glass substrate were 


\section{$(\mathrm{AuNPs} / \mathrm{PAH})_{3}$ Unannealing}
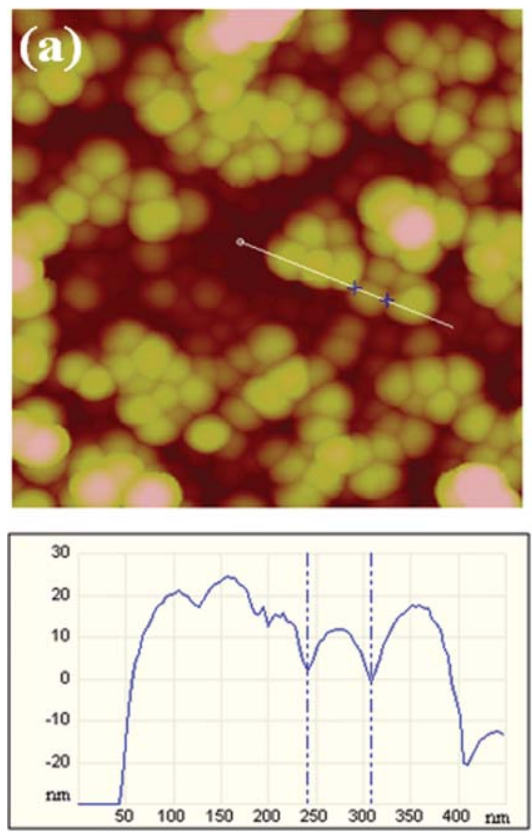

(AuNPs/PAH $)_{3}$ $300^{\circ} \mathrm{C} 1 \mathrm{~h}$
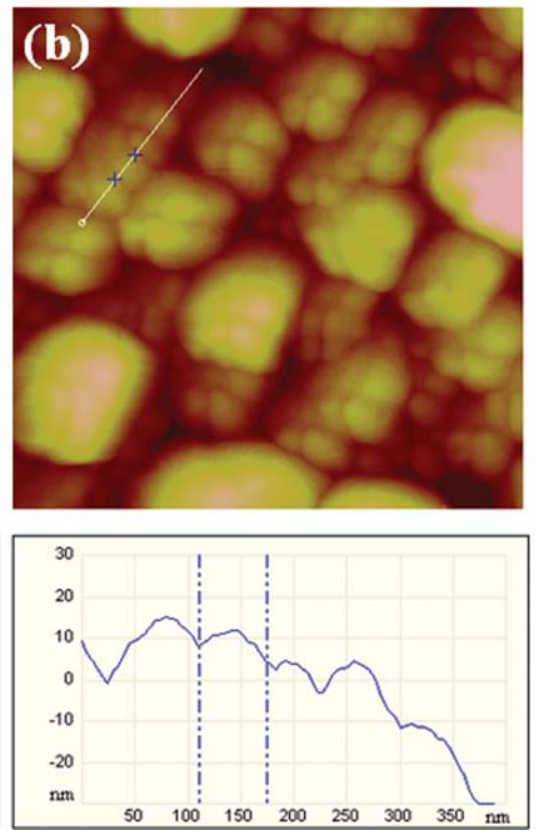

(AuNPs/PAH $)_{3}$ $500^{\circ} \mathrm{C} 1 \mathrm{~h}$
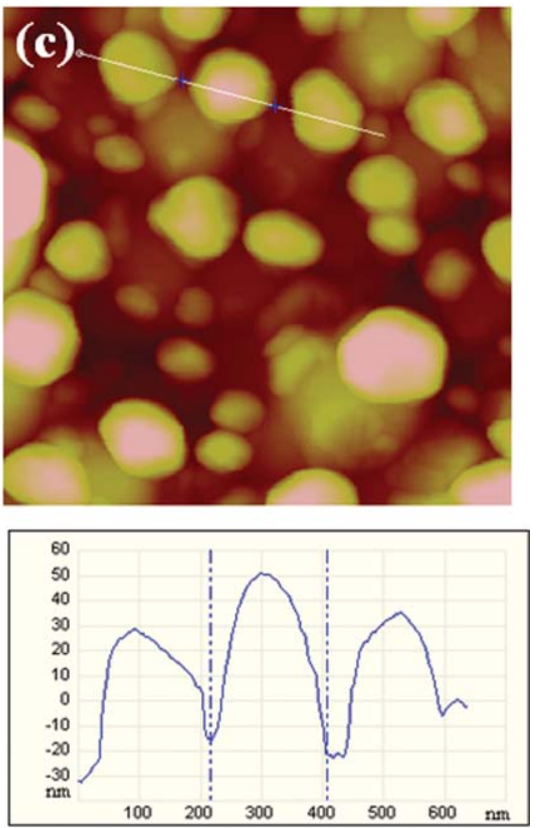

Fig. 3 AFM images of multilayer (AuNP/PAH) $)_{3}$ film $(1 \mu \mathrm{m} \times 1 \mu \mathrm{m})$ : (a) before annealing, (b) after annealing at $300^{\circ} \mathrm{C}$ for $1 \mathrm{~h}$, and (c) after annealing at $500{ }^{\circ} \mathrm{C} 1 \mathrm{~h}$. A typical cross-section of AuNPs is shown below each AFM images.

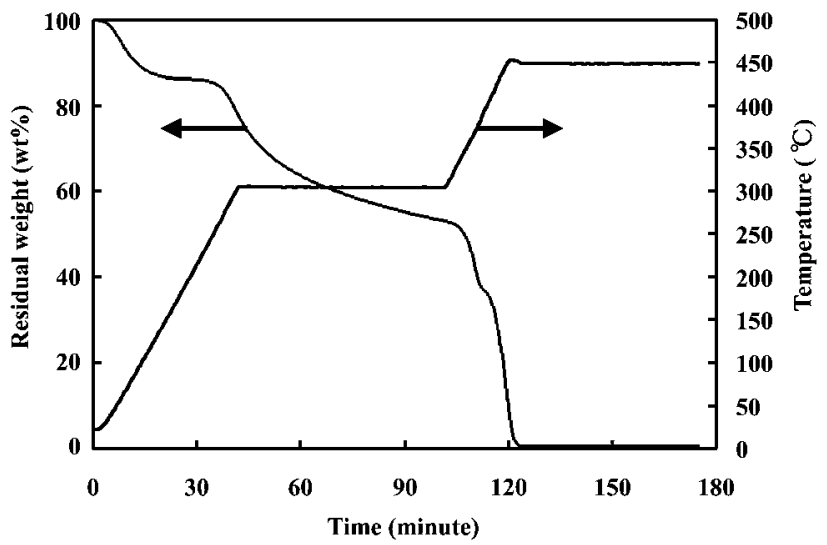

Fig. 4 TG curve of poly(allylamine hydrochloride) under air. After heating at $300{ }^{\circ} \mathrm{C}$ for $1 \mathrm{~h}$, arose the temperature to $450^{\circ} \mathrm{C}$ and maintained for $1 \mathrm{~h}$.

stable when immersed in organic solvents. The $(\mathrm{AuNP} / \mathrm{PAH})_{3}$ film after annealing at $300{ }^{\circ} \mathrm{C}$ (i.e. gold raspberry film) had higher stability compared to that after annealing at $500{ }^{\circ} \mathrm{C}$. The gold raspberry film was prepared at $300{ }^{\circ} \mathrm{C}$, and hence the cationic PAH polymer should still be presenting on the surface (or between nanoparticles in the raspberry films), as demonstrated in TG data of Fig. 4. The presenting PAH polymer can contribute to the high stability of the gold raspberry film. In a previous study, Isaacs et al. reported the high stability on thermal and ultrasonic treatments for a $(\mathrm{AuNP} / \mathrm{PAH})_{n}$ multilayer film. ${ }^{46}$

It should be also noted that the procedure requires gently handling of the AuNP-deposited substrate, since the detachment of AuNPs on the glass substrate may occur when rinsing with or immersing in various solvents. In measurements on LSPR wavelength shift in various solvents, the sequence in immersing the substrate in organic solvents was important. The immobilized AuNPs were stable, in the following sequence: benzaldehyde, benzene, glycerin, ethylene glycol, 1-propanol and finally water. Detachment of the AuNPs occurred in reverse order.

From the RIU values shown in Fig. 5d, it can be said that the highest LSPR sensitivity (i.e., the highest RIU) is obtained for the self-assembled (AuNP/PAH) $)_{3}$ film annealed at $300{ }^{\circ} \mathrm{C}$ for $1 \mathrm{~h}$; this film has a raspberry-like morphology. Herein, we performed the extraction and detection of Lyz by LSPR sensing using this AuNP film with a raspberry-like morphology. As Lyz is a high-pI enzyme that hydrolyzes the polysaccharide walls of bacteria, it is found to be widely distributed in body tissues and secretions. Increased Lyz concentrations in urine and serum have been recognized as being associated with leukemia, ${ }^{47}$ renal diseases, ${ }^{48}$ and meningitis. ${ }^{49}$ For the extraction-based approach, the AuNP film with raspberry-like morphology was functionalized with 11mercaptoundecanoic acid (MUA) to enable the binding of Lyz $(\mathrm{p} I=11)$ to the MUA-modified gold surface. The identical glass substrate (including annealing and MUA addition) was used for each measurement as shown in Fig. 6b, and the increase in the Lyz concentration was successively performed without washing process by adding a given concentration of Lyz into the specialized cell. We found that the relevant superior limit of detection (LOD) for the LSPR sensing of Lyz in a buffer solution was in the $10^{-11} \mathrm{~g} \mathrm{~mL}^{-1}\left(\sim 10^{-12} \mathrm{M}\right)$ range; this is shown in Fig. 6a. A slight LSPR wavelength shift was observed for picomolar-range concentrations of Lyz in the buffer solution. On the other hand, the use of multilayer AuNPs films, annealed at 
(a)

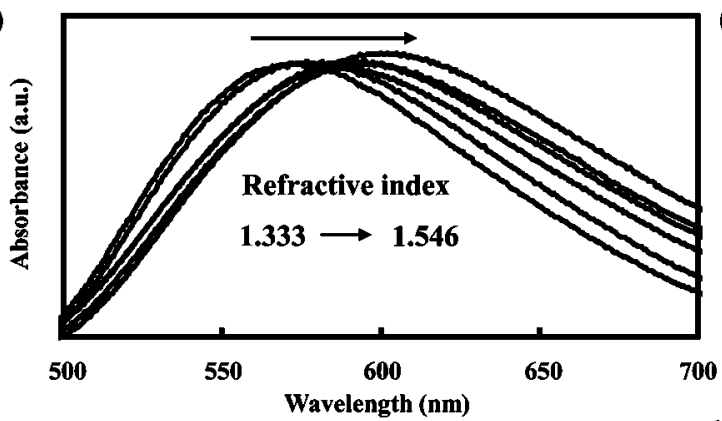

(b)

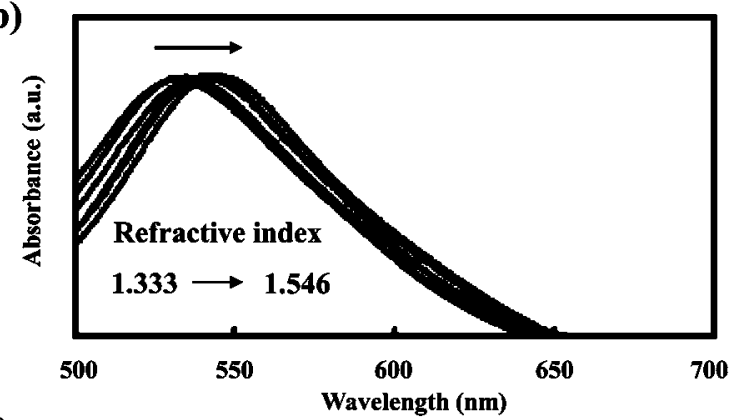

(c)

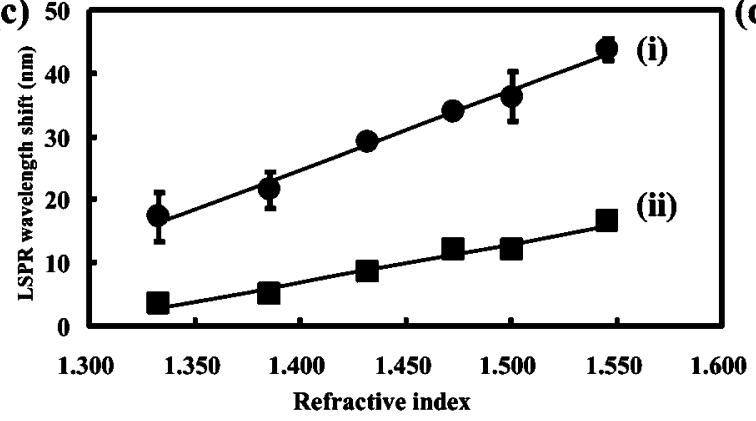

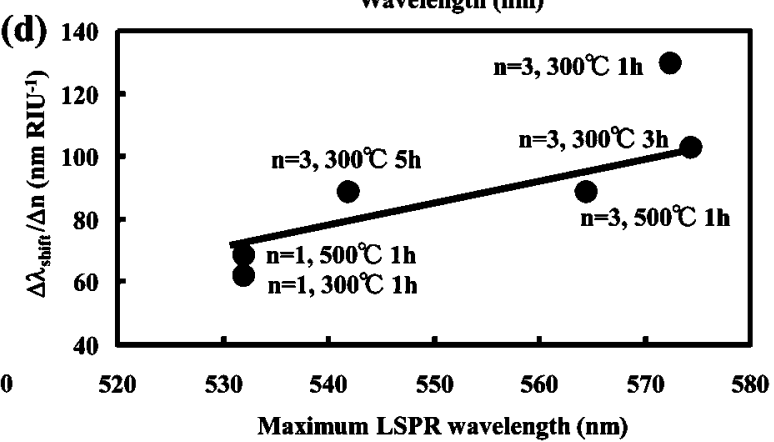

Fig. 5 UV-vis spectra of multilayer (AuNP/PAH) films after annealing at $300{ }^{\circ} \mathrm{C}$ for $1 \mathrm{~h}$, measured in various solvents, (a) $n=3$ and (b) $n=1$. (c) Dependence of LSPR wavelength shift $\left(\lambda_{\text {shift }} \text { ) on the refractive index for multilayer (AuNP/PAH) }\right)_{n}$ film: (i) $n=3$, after annealing at $300{ }^{\circ} \mathrm{C}$ for $1 \mathrm{~h}$, RIU value is $125 \mathrm{~nm}$ per RIU and (ii) $n=1$, after annealing at $300{ }^{\circ} \mathrm{C}$ for $1 \mathrm{~h}$, RIU value is $62 \mathrm{~nm}$ per RIU. (d) The relationship between the RIU value $\left(\Delta \lambda_{\text {shift }} / \Delta n\right)$ and the maximum LSPR wavelength.

$300{ }^{\circ} \mathrm{C}$ for a relatively longer duration of $5 \mathrm{~h}$ for which the raspberry-like morphology disappeared, was unsuitable even for detecting the concentration of Lyz $\left(10^{-9} \mathrm{~g} \mathrm{~mL}^{-1}\right)$ in the buffer solution (not shown). This result demonstrates that AuNP films with a raspberry-like morphology have a great potential for use in T-LSPR sensing.

In LSPR sensing, the RIU values depend on how confined the electromagnetic field associated with the LSPR is to the surface of the nanoparticle. ${ }^{1-9}$ The confinement of the electromagnetic field is enhanced by increasing the curvature of nanoparticles or reducing the distance between neighboring nanoparticles (i.e., by creating a hot spot).$^{1-9}$ It has been reported that these hot spots, where the field is most intense, such as the edge of the nanoplate and the gap between neighboring nanoparticles, contribute to the enhancement in LSPR sensitivity. The LSPR sensitivity depends on the location of the molecular binding on the metal nanostructures. ${ }^{50}$ For example, when the analyte molecules bind in the region of the hot spots, where the field is most intense, a larger LSPR wavelength shift is induced. In the case of the AuNP films with a raspberry-like morphology, the electromagnetic field
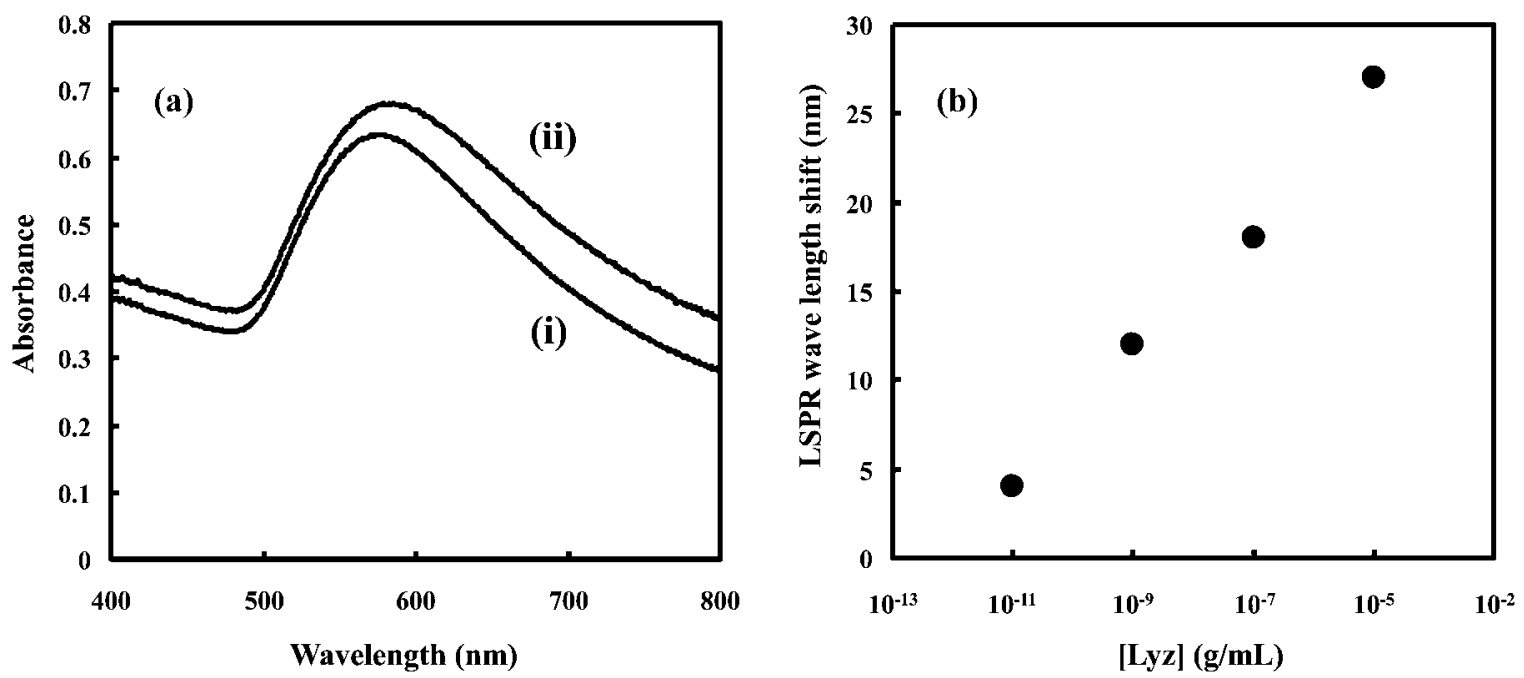

Fig. 6 (a) UV-vis spectra of multilayer (AuNP/PAH) $)_{3}$ films after annealing at $300^{\circ} \mathrm{C}$ for $1 \mathrm{~h}$ : (i) in buffer solution and (ii) in buffer solution containing $10^{-11} \mathrm{~g} \mathrm{~mL}^{-1}$ Lyz. (b) The limit of detection of multilayer (AuNP/PAH) 3 films after annealing at $300{ }^{\circ} \mathrm{C}$ for $1 \mathrm{~h}$. 
confinement can be enhanced in the gap among neighboring nanoparticles. The higher LSPR sensitivity observed for a raspberry-like assembly of AuNPs could be due to the preferential adsorption of Lyz into such gaps where the field is most intense, resulting in a larger LSPR wavelength shift. To confirm this possibility, we attempted the direct observation of Lyz in the gap among neighboring nanoparticles by AFM images. However, direct observation was impossible because of the large value of roughness of the nanoparticle film.

\section{Combination analysis of LSPR sensing and MALDI-MS for} detection of lysozyme in human serum

In a previous study, we demonstrated that the clustering of AuNPs enhanced the laser desorption/ionization (LDI) performance of peptides during the LDI-MS performed using AuNPs. ${ }^{51}$ Thus, the self-assembled AuNP films with a raspberrylike morphology are suitable to be used as the sample plate for LDI-MS. Next, we carried out the extraction, detection, and identification of Lyz in human serum using AuNP films with a raspberry-like morphology in the combination analysis of LSPR sensing and MALDI-MS. A schematic summary of the combination analysis of LSPR with MALDI-MS is shown in Fig. 7. Human albumin is the most abundant in proteins in human serum, and the suppression of albumin binding to nanoparticles is therefore, important for selectively detecting Lyz in human serum. ${ }^{52}$ Fig. 8a shows the UV-vis spectra of the MUAmodified AuNP film with a raspberry-like morphology before and after the immersion of the plate in Lyz $\left(10^{-4} \mathrm{~g} \mathrm{~mL}^{-1}\right)$-spiked human serum. After immersing the film, a large LSPR (a)

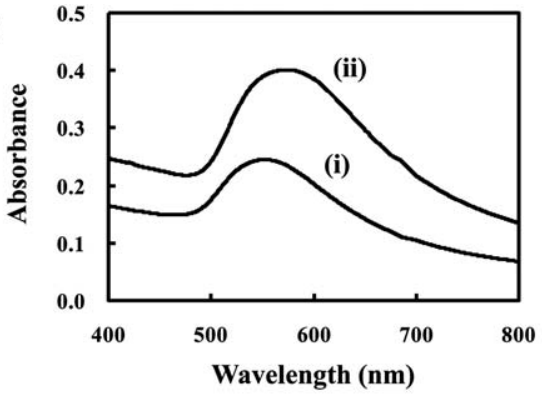

(b)

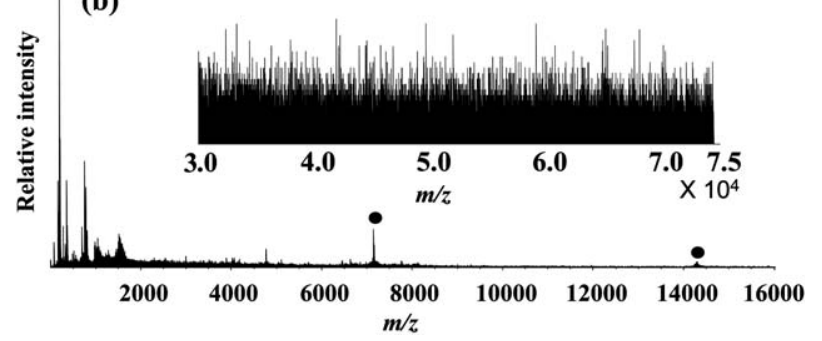

Fig. 8 (a) UV-vis spectra of raspberry-like assembly of AuNPs functionalized with MUA at air: (i) before immersing in human serum including Lyz $\left(10^{-4} \mathrm{~g} \mathrm{~mL}^{-1}\right)$ measured under air and (ii) after immersing in human serum including Lyz $\left(10^{-4} \mathrm{~g} \mathrm{~mL}^{-1}\right)$ measured under air. (b) MALDI mass spectrum of MUA-modified raspberry-like assembly of AuNPs after immersing in human serum including Lyz $\left(10^{-4} \mathrm{~g} \mathrm{~mL}^{-1}\right)$.

wavelength shift from 552 to $574 \mathrm{~nm}$ was observed. However, only the LSPR wavelength shift cannot be used to confirm the identification of Lyz because human serum contains various

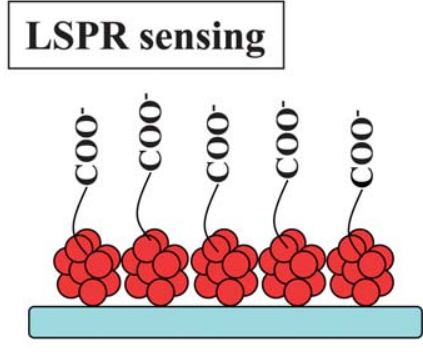

MUA-modified raspberry-like assembly of AuNPs

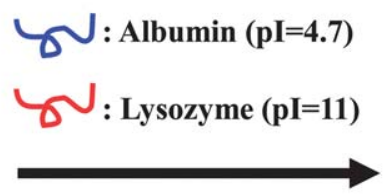

Immersing in human serum including $\operatorname{Lyz}\left(10^{-4} \mathrm{~g} / \mathrm{mL}\right)(\mathrm{pH}=8)$
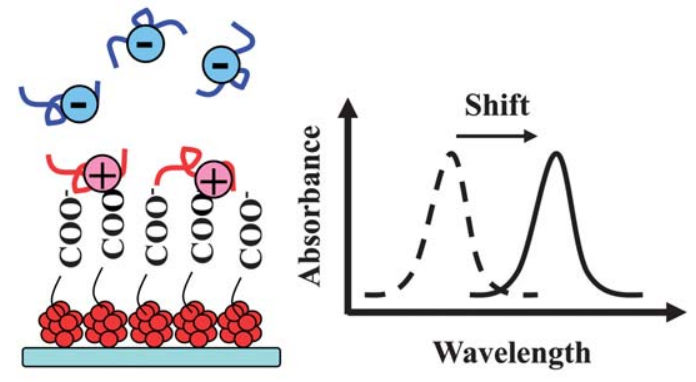

Wavelength

\section{MALDI-MS}
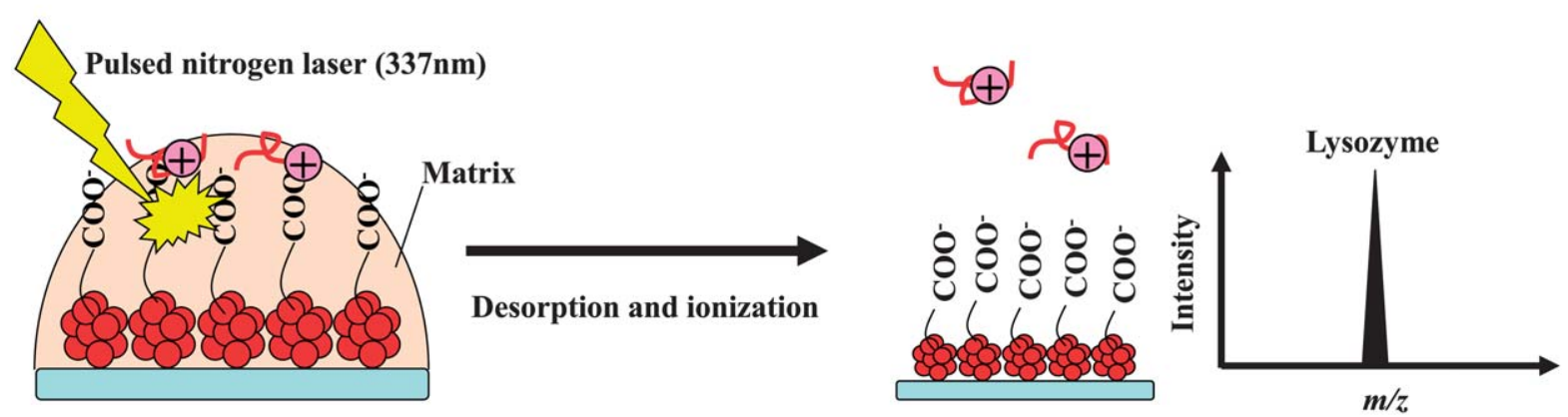

Fig. 7 Schematic summary of the combination analysis of LSPR sensing with MALDI-MS procedures. 
compounds such as human albumin and lipids that may bind with the AuNP film. Thus, a complementary analytical technique is required to identify the unknown molecules bound to the surface. For this purpose, the Lyz bound to the raspberry-like surface of the AuNPs was analyzed by MALDI-MS for the identification of Lyz in human serum. Fig. 8b shows the MALDI mass spectrum of the MUA-modified AuNP film with a raspberry-like morphology after the immersion of the plate in human serum containing Lyz $\left(10^{-4} \mathrm{~g} \mathrm{~mL}^{-1}\right)$. The MALDI mass spectrum shows molecular ions of Lyz at an $m / z$ of around 14 304, as well as at an $\mathrm{m} / \mathrm{z}$ of 7153 for multiple adducts (as denoted by closed circles). The molecular ion peaks from albumin were not detected in the mass range from 30000 to 75000 , indicating the successful extraction of Lyz in human serum by the MUA-modified AuNP film with a raspberry-like morphology. The MUA-modified gold surface is expected to detect Lyz in human serum through the electrostatic attraction between the positively charged Lyz and the negatively charged MUA in serum; as expected, the negatively charged MUA has very little interaction with the negatively charged albumin in human serum. These results demonstrate that a MUA-modified AuNP film with a raspberrylike morphology can be used in the combination analysis of LSPR sensing and MALDI-MS for the successful detection of Lyz in a complex mixture such as human serum.

In the serum sample spiked with Lyz, the detection sensitivity of Lyz by MALDI-MS was $10^{-4} \mathrm{~g} \mathrm{~mL}^{-1}$ in this methodology. The normal concentration of Lyz in serum is in the range of 5$10 \mu \mathrm{g} \mathrm{mL}^{-1}$, which is lower than the detection sensitivity in our system. Further increase in the sensitivity is, thus, necessary for the practical use of the LSPR/MALDI platform in this method.

\section{Conclusion}

Using aggregated AuNPs on an unannealed (AuNP/PAH) $)_{3}$ film as a template, we fabricated a film of self-assembled AuNPs with a raspberry-like morphology on a glass plate through the thermal annealing of a $(\mathrm{AuNP} / \mathrm{PAH})_{3}$ film at $300{ }^{\circ} \mathrm{C}$ for $1 \mathrm{~h}$. The plasmonic properties of the annealed AuNP films were controlled by changing the annealing temperature, duration of annealing, and the number of layers of the AuNPs. The AuNP film with raspberry-like morphology produced the highest refractive index unit (RIU) from among all the examined LbL films of AuNPs. The superior limit of detection (LOD) for the LSPR sensing of lysozyme in buffer solution was found to be in the picomolar range $\left(\sim 10^{-12} \mathrm{M}\right)$. At higher annealing temperatures and for longer durations of annealing, the raspberry-like morphology disappeared, and consequently, the LSPR sensitivity decreased. Further, we demonstrated the combination analysis of LSPR sensing and MALDI-MS for the selective detection and identification of lysozyme in human serum, which is a complex mixture, by using self-assembled AuNP films with a raspberrylike morphology.

\section{Acknowledgements}

This study was partially supported by a Grant-in-Aid for Scientific Research (B) (no. 22350040) from the Japan Society for the Promotion of Science (JSPS) and by the Core-to-Core Program promoted by JSPS (Project No. 18004). This study was also supported by the Strategic Project to Support the Formation of Research Bases at Private Universities of the Matching Fund Subsidy from the Ministry of Education, Culture, Sports, Science and Technology, Japan. We thank Ms Mai Kinoshita for helping the preparation of LbL films and LSPR sensing.

\section{References}

1 C. L. Haynes and R. P. Van Duyne, J. Phys. Chem. B, 2001, 105, 5599.

2 K. L. Kelly, E. Coronado, L. L. Zhao and G. C. Schatz, J. Phys. Chem. B, 2003, 105, 5599.

3 W. A. Murray and W. L. Barnes, Adv. Mater., 2007, 19, 3771.

4 A. M. Schwartzberg and J. Z. Zhang, J. Phys. Chem. C, 2008, 112, 10323.

5 M. E. Stewart, C. R. Anderton, L. B. Thompson, J. Maraia, S. K. Gray, J. A. Rogers and R. G. Nuzzo, Chem. Rev., 2008, 108, 494.

6 Y.-S. Shon, H. Y. Choi, M. S. Guerrero and C. Kwon, Plasmonics, 2009, 4, 95.

7 M. M. Miller and A. A. Lazarides, J. Phys. Chem. B, 2005, 109, 21556.

8 K.-S. Lee and M. A. El-Sayed, J. Phys. Chem. B, 2006, 110, 19220.

9 B. Sepủlveda, P. C. Angelomé, L. M. Lechuga and L. M. Liz-Marzàn, Nano Today, 2009, 4, 244.

10 T. Okamoto, I. Yamaguchi and T. Kobayashi, Opt. Lett., 2000, 25, 372.

11 N. Nath and A. Chilkoti, Anal. Chem., 2002, 74, 504.

12 A. J. Haes and R. P. Van Duyne, J. Am. Chem. Soc., 2002, 124, 10596.

13 F. Frederix, J.-M. Friedt, K.-H. Choi, W. Laureyn, A. Campitelli, D. Mondelaers, G. Maes and G. Borghs, Anal. Chem., 2003, 75, 6894.

14 H.-Y. Lin, C.-T. Chen and Y.-C. Chen, Anal. Chem., 2006, 78, 6873.

15 F. Tam, C. Moran and N. Halas, J. Phys. Chem. B, 2004, 108, 17290.

16 S. M. Marinakos, S. Chen and A. Chilkoti, Anal. Chem., 2007, 79, 5278.

17 W. B. Zhao, J. Park, A.-M. Caminade, S.-J. Jeong, Y. H. Jang, S. O. Kim, J.-P. Majoral, J. Chao and D. H. Kim, J. Mater. Chem., 2009, 19, 2006.

18 K. M. Mayer, S. Lee, H. Liao, B. C. Rostro, A. Fuentes, P. T. Scully, C. L. Nehl and J. H. Hafner, ACS Nano, 2008, 2, 687.

19 T. R. Jensen, M. L. Duval, K. L. Kelly, A. A. Lazarides, G. C. Schatz and R. P. Van Duyne, J. Phys. Chem. B, 1999, 103, 9846.

20 T. R. Jensen, M. D. Malinsky, C. L. Haynes and R. P. Van Duyne, $J$. Phys. Chem. B, 2000, 104, 10549.

21 C. L. Haynes and P. P. Van Duyne, J. Phys. Chem. B, 2001, 105, 5599.

22 M. D. Malinsky, K. L. Kelly, G. C. Schatz and R. P. Van Duyne, J. Phys. Chem. B, 2001, 105, 2343.

23 M. D. Malinsky, K. L. Kelly, G. C. Schatz and R. P. Van Duyne, J. Am. Chem. Soc., 2001, 123, 1471.

24 A. J. Haes, S. Zou, G. C. Schatz and R. P. Van Duyne, J. Phys. Chem. $B, 2004,108,109$.

25 A. J. Haes, S. Zou, G. C. Schatz and R. P. Van Duyne, J. Phys. Chem. $B, 2004,108,6961$.

26 A. V. Whitney, J. W. Elam, S. Zou, A. V. Zinovev, P. C. Stair, G. C. Schatz and R. P. Van Duyne, J. Phys. Chem. B, 2005, 109, 20522.

27 J. Zhao, A. Das, X. Zhang, G. C. Schatz, S. G. Sligar and R. P. Van Duyne, J. Am. Chem. Soc., 2006, 128, 11004.

28 J. Zhao, L. Jensen, J. Sung, S. Zou, G. C. Schatz and R. P. Van Duyne, J. Am. Chem. Soc., 2007, 129, 7647.

29 A. G. Brolo, R. Gordon, B. Leathem and K. L. Kavanagh, Langmuir, 2004, 20, 4813.

30 A. G. Brolo, S. C. Kwok, M. D. Cooper, M. G. Moffitt, C.-W. Wang, R. Gordon, J. Riordon and K. L. Kavanagh, J. Phys. Chem. B, 2006, 110, 8307.

31 A. D. Leebeeck, L. K. S. Kumar, V. de Lange, D. Sinton, R. Gordon and A. G. Brolo, Anal. Chem., 2007, 79, 4094.

32 J. C. Sharpe, J. S. Mitchell, L. Lin, N. Sedoglavich and R. Blaikie, Anal. Chem., 2008, 80, 2244.

33 G. Kalyuzhny, A. Vaskevich, G. Ashkenasy, A. Shanzer and I. Rubinstein, J. Phys. Chem. B, 2000, 104, 8238.

34 G. Kalyuzhny, M. A. Schneeweiss, A. Shanzer, A. Vaskevich and I. Rubinstein, J. Am. Chem. Soc., 2001, 123, 3177.

35 G. Kalyuzhny, A. Vaskevich, M. A. Schneeweiss and I. Rubinstein, Chem.-Eur. J., 2002, 8, 3850.

36 I. Doron-Mor, Z. Barkay, N. Filip-Granit, A. Vaskevich and I. Rubinstein, Chem. Mater., 2004, 16, 3476. 
37 I. Doron-Mor, H. Cohen, Z. Barkay, A. Shanzer, A. Vaskevich and I. Rubinstein, Chem.-Eur. J., 2005, 11, 5555.

38 I. Ruach-Nir, T. A. Bendikov, I. Doron-Mor, Z. Barkay, A. Vaskevich and I. Rubinstein, J. Am. Chem. Soc., 2007, 129, 84.

39 T. Karakouz, D. Holder, M. Goomanovsky, A. Vaskevich and I. Rubinstein, Chem. Mater., 2009, 21, 5875.

40 J. N. Anker, W. P. Hall, M. P. Lambert, P. T. Velasco, M. Mrksich, W. L. Klein and R. P. Van Duyne, J. Phys. Chem. C, 2009, 113, 5891.

41 H. Kawasaki, T. Sugitani, T. Watanabe, T. Yonezawa, H. Moriwaki and R. Arakawa, Anal. Chem., 2008, 80, 7524.

42 (a) S. Sun, C. B. Murray, D. Weller, A. Folks and A. Moser, Science, 2000, 287, 1989; (b) K. E. Elkins, T. S. Vedantam, J. P. Liu, H. Zeng, S. Sun, Y. Ding and Z. L. Wang, Nano Lett., 2003, 3, 1647.

43 X. Hu, T. Wang and S. Dong, J. Colloid Interface Sci., 2007, 316, 647.

44 H. Kawasaki, K. Nishimura and R. Arakawa, J. Phys. Chem. C, 2007, 111, 2683.
45 J. C. Maxwell-Granett, Philos. Trans. R. Soc. London, Ser. A, 1904, 203, 385.

46 S. R. Isaacs, H. Choo, W.-B. Ko and Y.-S. Shon, Chem. Mater., 2006, 18, 107.

47 S. S. Levinson, R. J. Elin and L. Yam, Clin. Chem. (Washington, DC, U. S. ), 2002, 48, 1131.

48 J. F. Harrison, G. S. Lunt, P. Scott and J. D. Blainey, Lancet, 1968, 1, 371.

49 M. Klockars, S. Reitamo, T. Weber and Y. Kerttula, Acta Med. Scand., 1978, 203, 71.

50 S. R. Beeram and F. P. Zamborini, J. Am. Chem. Soc., 2009, 131, 11689.

51 A. Tarui, H. Kawasaki, T. Taiko, T. Watanabe, T. Yonezawa and R. Arakawa, J. Nanosci. Nanotechnol., 2009, 9, 159.

52 H. Kawasaki, T. Akira, T. Watanabe, K. Nozaki, T. Yonezawa and R. Arakawa, Anal. Bioanal. Chem., 2009, 395, 1423. 